\title{
Rolado selectivo de baja intensidad (RBI) sobre bosque nativo en el Chaco Semiárido: influencia sobre la diversidad y la composición de un ensamble de reptiles
}

\author{
Rubén D. Coria ${ }^{\varpi}$; Carlos R. Kunst \& Víctor Navarrete \\ InstitutoNacionaldeTecnologíaAgropecuaria,EstaciónExperimentalAgropecuariaSantiagodelEstero.SantiagodelEstero, \\ Argentina.
}

\begin{abstract}
RESUMEN. El rolado selectivo de baja de intensidad (RBI) es una tecnología para habilitar sistemas silvopastoriles de bajo impacto en el Chaco Semiárido. A diferencia de otras formas de habilitar las tierras con fines ganaderos (e.g., desmonte total, rolados intensivos), el RBI conserva bien el suelo y la mayor parte de la vegetación nativa, sin resignar producción. Este estudio aborda a escala local la influencia del RBI sin siembra de pasturas exóticas sobre un ensamble de reptiles de un bosque nativo del Chaco Semiárido, donde el bosque con RBI mantiene conectividad con vastas superficies de vegetación nativa.Los resultados muestran que: a) la diversidad alfa de reptiles no disminuyó en el bosque con RBI debido aque la estructura vertical de la vegetación no fue degradada, y b) el ensamble de reptiles del bosque con RBI fue muy parecido al del bosque testigo dada la gran similitud estructural entre estos hábitats. Los resultados demuestran que la cobertura arbórea y de hojarasca son variables importantes de estructura de la vegetación que influirían sobre la distribución por hábitat de las especies de reptiles, relacionadas con un gradiente ambiental de luz solar. Este estudio sugiere que localmente, el RBI sin siembra de pasturas exóticas tiene potencial para conservar de manera adecuada la diversidad y la composición de los ensambles de reptiles de bosques nativos del Chaco Semiárido.
\end{abstract}

[Palabras clave: diversidad alfa, similitud de ensambles, estructura de la vegetación, similitud estructural de hábitats, sistemas silvopastoriles]

\begin{abstract}
Low intensity roller chopping (RBI) on forests of Semiarid Chaco: influence on diversity and composition of a reptile assemblage. Low intensity roller chopping (RBI) is a technology that enables low impact silvopastoral systems in the Semiarid Chaco. In contrast to other systems, RBI preserves the soil and most of the native vegetation without sacrificing production. This study addresses the local impact of RBI without implanted exotic pastures on a reptile assemblage in a Semiarid Chaco forest, where the forest with RBI maintains connectivity with large areas of native vegetation. The results show that: a) the alpha diversity of reptiles did not decrease in the forest with RBI, associated with the non-degradation of the vegetation's vertical structure, and b) reptile assemblages were highly similar between the forest with RBI and the control, due to a high structural similarity between habitats. The results show that tree and litter cover are important variables that influence the distribution of reptile species in the habitats, related to the sunlight gradient. At a local scale, this study suggests that RBI without implanted exotic pastures has the potential to adequately maintain the diversity and composition of reptile assemblages in Semiarid Chaco forests.
\end{abstract}

[Keywords: alpha diversity, similarity of assemblages, vegetation structure,habitat structural similarity, silvopastoral systems]

\section{INTRODUCCIÓN}

El rolado es una práctica muy utilizada en el Chaco Semiárido para crear sistemas silvopastoriles. El rolado consiste en aplastar y triturar la vegetación nativa con una herramienta denominada rolo, que es un cilindro metálico hueco de más de $2 \mathrm{~m}$ de ancho y entre 1.2 y $1.5 \mathrm{~m}$ de diámetro. Lastrado con agua alcanza a pesar entre 3000 y $4000 \mathrm{~kg}$. Puede ser traccionado por tractores o topadoras de distinta potencia (Albanesi et al. 2013). Los rolados intensivos (i.e., los realizados con maquinarias de gran porte) tienen un enfoque exclusivamente ganadero y provocan

Editor asociado: Pedro Blendinger

$\triangle$ rudacoria@yahoo.com.ar gran impacto en el suelo y en la vegetación nativa, por lo que no son recomendables (Albanesi et al. 2013; Kunst et al. 2015a, b). A diferencia de los rolados intensivos, el rolado selectivo de baja intensidad (RBI) permite crear sistemas silvopastoriles de bajo impacto. El RBI se caracteriza por: a) emplear maquinarias pequeñas (i.e., ancho de rolo $<2.5 \mathrm{~m}$, tractores agrícolas), b) concentrar el disturbio en los arbustos y mantener la mayor parte de la estructura y composición de la vegetación nativa, c) mantener la estructura y la fertilidad del suelo, d) ser compatible con el aprovechamiento forestal, e) ser

Recibido: 16 de marzo de 2016

Aceptado: 25 de julio de 2016 
generalmente acompañado de la siembra de pasturas exóticas (e.g., Megathyrsus maximus cv. gatton panic) para mejorar la oferta forrajera, y f) utilizar la teoría de disturbios para planificar la intensidad, severidad, frecuencia y extensión de los tratamientos (Kunst et al. 2008; Albanesi et al. 2013; Kunst et al. 2015a,b). Los beneficios productivos y ambientales del RBI influyeron para que esta tecnología se difundiera con fuerza entre las provincias del Chaco Argentino (Coria et al. 2015).

Aún se conoce poco sobre los efectos del RBI sobre la fauna silvestre, y todos los estudios disponibles se realizaron en una misma área de estudio, el Campo Experimental F. Cantos, del INTA EEA Santiago del Estero (ex Campo Experimental La María), que también fue utilizada para este trabajo. El grupo más estudiado fue el de las aves (Coria et al. 2015), mientras que la información existente sobre los reptiles y anfibios es escasa (Kunst et al. 2016). En referencia a las aves, Coria et al. (2015) sugieren que el RBI no disminuye la diversidad alfa y mantiene baja la diversidad beta de los ensambles debido a que genera hábitats similares en flora y estructura a los bosques sin disturbar. En otros sistemas silvopastoriles de bajo impacto del Chaco Semiárido (pero no creados mediante el RBI), al compararlos con fragmentos de bosques tampoco disminuye la diversidad alfa de aves y la similitud de los ensambles es alta (Mastrangelo and Gavin 2012). Kunst et al. (2016) reportaron que la diversidad alfa de reptiles y anfibios no disminuyó en un bosque tratado con RBI sin siembra de pasturas exóticas y de un año de edad, y lo atribuyeron al bajo impacto de esta tecnología sobre la vegetación. Pero estos autores no analizaron la composición de especies ni las relaciones entre los ensambles y las variables ambientales, que sí son abordadas en este estudio. Cabe señalar que otros trabajos reportan una influencia de los cambios en la vegetación nativa sobre los ensambles de reptiles (Leynaud and Bucher 2005; Pelegrin et al. 2009; Pelegrin and Bucher 2010, 2012) y de anfibios (Lescano et al. 2015) en el Chaco Seco.

El presente estudio aborda a escala local la influencia del RBI sin siembra de pasturas exóticas sobre un ensamble de reptiles de un bosque nativo del Chaco Semiárido, donde el bosque con RBI evaluado se caracteriza por mantener conectividad con vastas superficies de vegetación nativa. Puntualmente, pretende dar respuesta a dos interrogantes.
El primero: ¿cuál es la relación entre el RBI y la diversidad alfa de reptiles? La hipótesis de la heterogeneidad de los hábitats sostiene que los hábitats estructuralmente complejos pueden proveer más nichos y diversidad de maneras de explotar los recursos ambientales y, por lo tanto, incrementan la diversidad de especies (Tews et al. 2004; Fischer et al. 2006). En este estudio se espera que el RBI conserve la estructura de la vegetación del bosque nativo $y$, vinculado a ello, que no disminuya significativamente la diversidad alfa de reptiles (predicción 1). El segundo interrogante a evaluar es la relación entre el RBI y la composición del ensamble de reptiles. Según Fischer et al. (2006), las posibilidades de utilización de los hábitats antropizados por parte de las especies animales están influenciadas por las similitudes estructurales entre esos hábitats y los hábitats naturales de referencia. A mayor similitud estructural, mayores posibilidades de utilización dado que los efectos de borde disminuyen, la conectividad se incrementa y hay disponibilidad de hábitats permanentes. En el Chaco Semiárido, estudios recientes a escala local mostraron que los hábitats más similares albergan los ensambles de aves más similares (Coria et al. 2015; Coria et al. 2016), lo cual es consistente con lo expuesto anteriormente. En este estudio se espera que el bosque con RBI sea estructuralmente similar al bosque sin disturbar y, en relación con ello, que las composiciones de los ensambles de reptiles sean también similares entre estos hábitats (predicción 2).

\section{Materiales y Métodos}

\section{Área de estudio}

El estudio se realizó en el Campo Experimental F. Cantos (ex Campo Experimental La María) (28 $\left.8^{\circ} 3^{\prime} \mathrm{S}-64^{\circ} 15^{\prime} \mathrm{O}\right)$ del INTA, Estación Experimental Agropecuaria Santiago del Estero, Provincia de Santiago del Estero, Argentina (Figura 1). El clima es semiárido subtropical con estacionalidad bien marcada (Boletta 1998). La temperatura promedio anual es $21^{\circ} \mathrm{C}$, con una mínima y una máxima media de 14 y $28^{\circ} \mathrm{C}$, respectivamente. La precipitación media es $574 \mathrm{~mm} / \mathrm{año}$, concentrada en los meses de octubre a marzo. Enclavado en la ecorregión Chaco Seco, subregión Chaco Semiárido (Morello et al. 2012), el Campo F. Cantos posee una matriz de vegetación nativa continua de $60 \mathrm{~km}^{2}$. El área de estudio mantiene conectividad con 


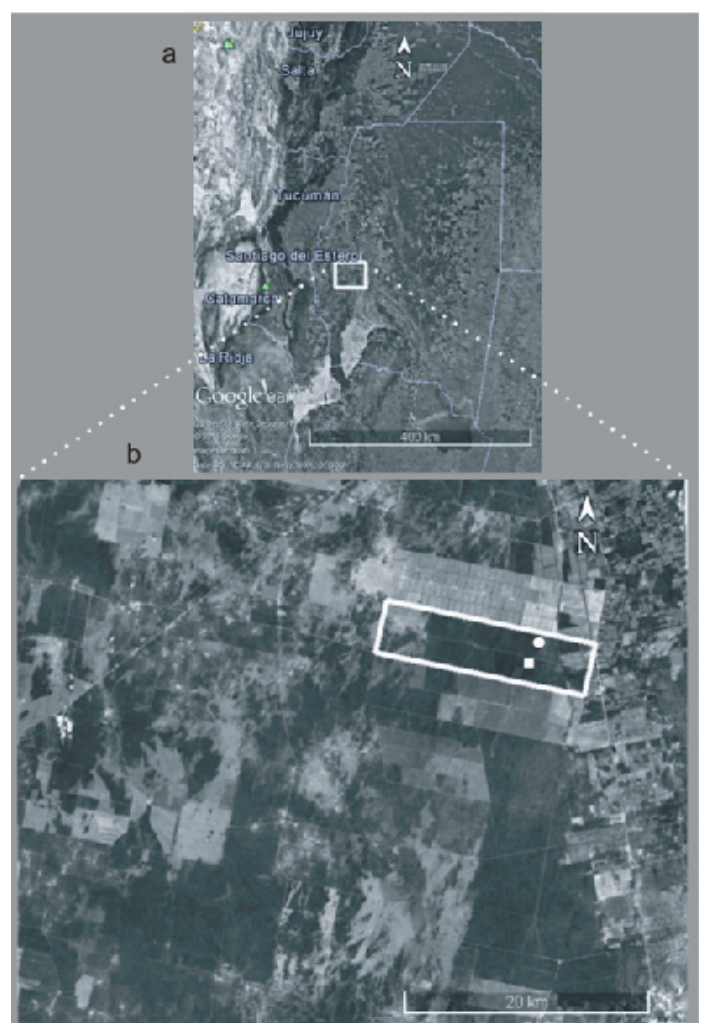

Figura 1. A) Ubicación del área de estudio en el contexto regional del norte de Argentina. B) Ampliación de la zona de influencia del Campo Experimental F. Cantos (rectángulo blanco), posición central del Bosque Testigo (punto cuadrangular blanco) y posición central del Bosque con RBI (punto circular blanco). Bosque Testigo: bosque con uso ganadero y sin aprovechamiento forestal intensivo durante 35 años; Bosque con RBI: bosque de 100 ha tratado con RBI, de cuatro años de antigüedad, sin siembra de pasturas exóticas, con pastoreo ganadero y corta forestal. Fuente de las imágenes: Google Earth Pro Versión 7.1.5.1557.

Figure 1. A) Location of study area in the regional context of northern Argentina. B) Influence zone of Campo Experimental F. Cantos (white rectangle), central location of Untreated Forest (white square point) and central location of Forest with RBI (white circular point). Untreated Forest: forest with cattle use without intense logging for 35 years; Forest with RBI: 100 ha forest treated with RBI, four years old, with unseeded exotic pastures, grazing livestock and logging. Images Source: Google Earth Pro Version 7.1.5.1557.

vastas superficies de vegetación nativa hacia el oeste y el sur, mientras que hacia el norte y el este predomina una matriz agrícola (Figura 1). La vegetación original del área de estudio se distribuía a lo largo de un gradiente de relieve y suelos: a) sitios altos, con suelos de texturas más gruesas y comunidad original de bosque, b) sitios bajos, con suelos de texturas más finas y representados originalmente por sabanas y pastizales, y c) sitios de medias lomas, con ecotonos entre los sitios anteriores, cuya comunidad original era una matriz de pastizal con árboles o isletas de bosques dispersos (Kunst et al. 2006; Kunst et al. 2015a). Estos sitios se encuentran a $1-2 \mathrm{~km}$ de distancia, y por el uso ganadero y forestal del lugar, las comunidades originales se hallan modificadas:los sitios altos están ocupados por bosques de quebrachos densamente arbustizados; los sitios bajos, por sabanas de Elionurus muticus (Aibe) y arbustales más o menos densos, y los sitios de medias lomas, por ecotonos arbustizados. En los sectores de bosque, los árboles dominantes son Aspidosperma quebracho-blanco (quebracho blanco) y Schinopsis lorentzii (quebracho colorado), seguidos por Ziziphus mistol (mistol) y Prosopis nigra (algarrobo negro). Los arbustos dominantes son Acacia gilliesii, CapparisatamisqueayCeltisehrenbergiana,con menor participación de Moya spinosa. En el estrato herbáceo son frecuentes las gramíneas Gouinialatifolia, Gouniaparaguayensis, Setaria lachnea, Setaria sp. y Trichloris crinita, y entre las latifoliadas, Justicia squarrosa, Wissadula densiflora y Amphilophium carolinae.

\section{Tipos de hábitats}

Los hábitats estudiados se ubicaron en los sectores de bosque de los sitios altos: a) Bosque Testigo: bosque con uso ganadero $\mathrm{y}$ sin aprovechamiento forestal intensivo durante 35 años, y b) Bosque con RBI: bosque de 100 ha tratado con RBI, de cuatro años de antigüedad, sin siembra de pasturas exóticas, con uso ganadero y corta forestal. El disturbio inicialmente aplicado fue planificado para no reducir la cobertura arbustiva más allá del $30 \%$ por unidad de superficie ni el área basal de árboles más allá de $6 \mathrm{~m}^{2} / \mathrm{ha}$, y fue considerado como de intensidad media a baja por la acción combinada del rolado y la corta forestal. Desde la aplicación inicial del disturbio al momento de la realización del presente estudio la vegetación no fue re-disturbada con rolado y corta forestal, y sólo se realizó pastoreo ganadero con una frecuencia de entrada de animales de una vez por año.

\section{Muestreo de reptiles}

Las unidades de muestreo fueron parcelas circulares de $50 \mathrm{~m}$ de radio. Por cada hábitat se ubicaron sistemáticamente 7 unidades de muestreo, dando un total de 14. Los puntos centrales de cada unidad se ubicaron a más de $250 \mathrm{~m}$ entre ellos (Leynaud and Bucher 2005) y a 200-300m de los bordes de caminos internos. La abundancia por especie de reptiles se midió con trampas cerco-pozo (adaptado de Cano and Leynaud 2009). En el centro de cada unidad de muestreo se ubicó una trampa 
que consistía en 4 recipientes enterrados a ras del suelo, con 3 recipientes satélites ubicados a $5 \mathrm{~m}$ de un recipiente central, con un ángulo de separación de $120^{\circ}$. Entre cada recipiente satélite y el recipiente central se colocó un cerco de tela media sombra de $50 \mathrm{~cm}$ de alto. Cada recipiente tuvo las siguientes dimensiones: volumen $=30 \mathrm{~L}$, diámetro $=33 \mathrm{~cm}$ y profundidad $=47 \mathrm{~cm}$. El principio de funcionamiento de la trampa es que los animales son conducidos por los cercos hacia algún recipiente donde caen y quedan atrapados. Para prevenir muertes de animales por ahogamiento en agua de lluvia y calor se construyeron drenes en cada recipiente y se cubrió el fondo con una densa capa de pastos secos. Las trampas permanecieron activas sin interrupción desde el 6/11/2015 al 09/ 12/2015, 34 días en total. Este período fue seleccionado para coincidir con los meses de mayor actividad de los reptiles (Fitzgerald et al. 1999; Richard 1999; Leynaud and Bucher 2001). Las trampas se revisaron a intervalos de aproximadamente 3 días. Los individuos capturados se marcaron con pintura esmalte blanco y se liberaron para evitar conteos dobles. No incluimos en los índices de diversidad y análisis estadísticos dos individuos correspondientes a dos especies posiblemente submuestreadas con las trampas cerco-pozo: Tropidurus spinulosus por ser de hábitos arbóreos (Leynaud and Bucher 2005) y Tupinambisrufescensqueporsutamaño(>1m de longitud como adulto) pudo escapar de las trampas.

\section{Muestreo de vegetación}

En cada unidad de muestreo se midió cobertura arbórea, arbustiva, herbácea, de residuos leñosos finos, residuos leñosos gruesos y de hojarasca. Las leñosas $>3 \mathrm{~m}$ de altura se consideraron cobertura arbórea y las $3 \mathrm{~m}$ de altura, cobertura arbustiva (Morello and Adamoli 1968; Kunst et al. 2006). Los residuos leñosos fueron materiales leñosos muertos dispuestos en el suelo, donde los gruesos fueron $>10 \mathrm{~cm}$ de diámetro y $>60 \mathrm{~cm}$ de longitud, y los finos, $<10 \mathrm{~cm}$ de diámetro o $<60 \mathrm{~cm}$ de longitud (Riffell et al. 2011). Los métodos utilizados fueron: a) coberturas arbórea y arbustiva (mediante el método de transecta de puntos [Riney 1982]. En cada unidad de muestreo se trazaron dos transectas de $45 \mathrm{~m}$ donde relevamos 30 puntos en cada una), b) coberturas de herbáceas, hojarasca, residuos leñosos gruesos y de residuos leñosos finos (mediante estimación visual en marco metálico de $0.25 \mathrm{~m}^{2}$, con seis submuestras por unidad de muestreo).

\section{Análisis de datos}

Para evaluar la predicción 1 se realizaron comparaciones entre hábitats de la complejidad estructural de la vegetación y de las riquezas de especies de reptiles. Por cada unidad de muestreo se obtuvieron seis variables de cobertura de la vegetación: a) cobertura arbórea (\%) y b) cobertura arbustiva (\%) (Riney 1982), c) cobertura herbácea (\%), d) cobertura de hojarasca (\%), e) cobertura de residuos leñosos gruesos (\%), y f) cobertura de residuos leñosos finos (\%). A partir de estas variables se obtuvieron dos medidas de la complejidad vertical de la vegetación: 1) riqueza estructural (i.e., número de coberturas vegetales presentes, rango 0-6), y 2) diversidad estructural (mediante el Índice de ShannonWeaver,

$$
\mathrm{H}=\sum_{\mathrm{i}}^{S} \mathrm{p}_{\mathrm{i}} \ln \mathrm{p}_{\mathrm{i}}
$$

donde $\mathrm{p}_{\mathrm{i}}=$ proporción de la cobertura vegetal i (Tews et al. 2004). Las diferencias significativas entre estas variables dependientes se evaluaron mediante la prueba $t$ con un nivel de significación $\alpha=0.1$ y tipos de hábitats como factor de clasificación. Para muestras pequeñas, como en este estudio, un valor de $\alpha$ más grande incrementa la potencia de la prueba estadística, lo cual es deseable (Di Rienzo et al. 2008). Se aplicó la transformación logarítmica a la matriz de datos para poder sostener los supuestos de normalidad y homogeneidad de varianzas de los residuos: $\left(b_{i j}=\log \left[x_{i j}+1\right]\right)$, donde $x_{i j}=$ valor original de la fila i y la columna $j$ y $b_{i j}=$ valor $x_{i j}$ transformado (Zar 1999). Se utilizó el Software INFOSTAT versión 2013 (Di Rienzo et al. 2013).

El número total de individuos capturados (individuos/trampa en 34 días) por especie de reptiles en cada unidad de muestreo se utilizó para obtener la riqueza observada de especies por hábitat, estimar la riqueza esperada de especies por hábitat y comparar estadísticamente la riqueza de especies entre hábitats. Se calculó el estimador no paramétrico Chao1 de la riqueza de especies (basado en el número de especies raras de la muestra) y su intervalo de confianza al 95\% (Colwell 2013). La comparación de la riqueza de especies entre hábitats se realizó mediante curvas de rarefacción utilizando el estimador Mao Tau, donde el solapamiento de los intervalos de confianza $(95 \%)$ de la riqueza esperada de especies fue utilizado como criterio de inferencia estadística al nivel de $\alpha=0.05$ (la falta de solapamiento indica diferencias no significativas) (Colwell 2013). El 
estimador Chao1 y las curvas de rarefacción se obtuvieron mediante el Software EstimateS 9.1.0 (Colwell 2013).

Para evaluar la predicción 2 se analizaron las similitudes estructurales de la vegetación y de los ensambles de reptiles entre hábitats. La similitud estructural de la vegetación se evaluó mediante la distancia de Bray-Curtis (BC), muy utilizada para el estudio de comunidades ecológicas (McCune et al. 2002). Para el cálculo de BC se utilizó la matriz con las ocho variables de estructura de la vegetación mencionadas anteriormente. Para mejorar el desempeño de BC previo al cálculo se relativizó la matriz de datos por columnas $\left(\mathrm{b}_{\mathrm{ii}}=\mathrm{x}_{\mathrm{ij}} / \mathrm{xmax}_{\mathrm{i}}\right)$, donde $\mathrm{x}_{\mathrm{ij}}=$ valor original de la fila i y la columna $j$, $x x^{11}=x_{i}=$ mayor valor de la columna j $b_{\mathrm{ii}}=$ valor $x_{\mathrm{ij}}$ transformado (McCune et al. 2002). BC se obtuvo mediante el Software INFOSTAT versión 2013 (Di Rienzo et al. 2013) y los valores se expresaron como porcentaje de similitud (S) mediante (1-BC)x100.

El número total de individuos capturados por especie de reptiles por unidad de muestreo se utilizó para obtener el estimador de la similitud de los ensambles Chao-Jaccard basado en la abundancia de especies (CH-J), el cual deriva del índice de similitud de Jaccard y considera la abundancia proporcional de las especies (Chao et al. 2005). Este estimador varía entre 0 y 1 , donde 0 indica total disimilitud y 1 total similitud entre dos ensambles. Incorpora el efecto de las especies no vistas, lo que disminuye el sesgo por el tamaño de la muestra y mejora su utilidad para inferir la similitud de los ensambles (Chao et al. 2005). El estimador CH-J se obtuvo mediante el Software EstimateS 9.1.0 (Colwell 2013).

Para explorar las relaciones entre las especies de reptiles y las variables de estructura de la vegetación se realizó un ordenamiento de las unidades de muestreo según el número total de individuos por especie. Para esto se usó la técnica de escalamiento multidimensional no métrico (NMS, siglas en inglés). El NMS es un procedimiento iterativo que minimiza la diferencia (estrés) entre la distancia en la matriz original y la distancia en el espacio de ordenación reducido. El número óptimo de ejes en una solución se determina a través del mínimo estrés y la prueba de significación de Monte Carlo (Clarke and Warwick 2001; McCune et al. 2002). Se utilizó la medida de distancia de Bray-Curtis (BC) sobre una matriz de datos con transformación logarítmica. Se construyó un gráfico Join-Plot para representar a las especies en el espacio de ordenación y establecer las relaciones con las variables ambientales fuertes $\left(\mathrm{r}^{2} 0.20\right)$ (McCune and Mefford 2011). Se utilizó el Software PC-ORD 6.15 (McCune and Mefford 2011).

\section{Resultados}

Se registraron 261 individuos: 107 en el Bosque Testigo y 154 en el Bosque con RBI (Tabla 1). Hubo seis especies de lagartos, una de tortuga y tres de serpientes. Las especies de lagartos Homonota fasciata, Leiosaurus paronae, Sternocercus doellojuradoi, Teius teyou y Tropidurus etheridgei fueron las más abundantes y representaron el $97.3 \%$ de los individuos capturados.

\section{Complejidad estructural de la vegetación y diversidad alfa de reptiles}

La cobertura arbórea y la arbustiva fueron, respectivamente, 20.4 y $37.0 \%$ menores en el bosque con RBI. La cobertura de residuos leñosos finos y gruesos fueron 184.8 y $348.4 \%$, respectivamente, mayores en el bosque con RBI. La cobertura de hojarasca y herbácea y la riqueza estructural no variaron entre tipos de bosque. La diversidad estructural fue mayor en el bosque con RBI (Tabla 2).

La riqueza de especies de reptiles en el Bosque Testigo fue 7, mientras que el estimador Chao1

Tabla 1. Abundancias de especies de reptiles (individuos/ trampa en 34 días) en dos tipos de bosque del Chaco Semiárido (media $\pm D E$; Bosque Testigo [ $n=7$ ] y Bosque con RBI [n=7]).Ver descripción de los tipos de bosque en la leyenda de la Figura 1.

Table 1. Abundances of reptile species (individuals/trap in 34 days) in two types of Semiarid Chaco forests (mean \pm SD; Untreated Forest [n=7] and Forest with RBI [n=7]). See description of forest types in the legend of Figure 1.

\begin{tabular}{|c|c|c|c|}
\hline Especie & Grupo & $\begin{array}{l}\text { Bosque } \\
\text { Testigo }\end{array}$ & $\begin{array}{c}\text { Bosque con } \\
\text { RBI } \\
\end{array}$ \\
\hline Chelonoidischilensis & Tortuga & & $0.1 \pm 0.4$ \\
\hline Homonota borellii & Lagarto & & $0.1 \pm 0.4$ \\
\hline Homonota fasciata & Lagarto & $2.0 \pm 1.3$ & $1.7 \pm 1.4$ \\
\hline Leiosaurus paronae & Lagarto & $0.9 \pm 0.9$ & $0.7 \pm 0.8$ \\
\hline $\begin{array}{l}\text { Siagonodonun } \\
\text { guirostris }\end{array}$ & Serpiente & & $0.3 \pm 0.5$ \\
\hline Xenodon pulcher & Serpiente & $0.3 \pm 0.5$ & \\
\hline Phimophis vittatus & Serpiente & $0.1 \pm 0.4$ & \\
\hline $\begin{array}{l}\text { Sternocercus } \\
\text { doellojuradoi }\end{array}$ & Lagarto & $3.0 \pm 2.1$ & $1.7 \pm 1.7$ \\
\hline Teius teyou & Lagarto & $8.9 \pm 6.0$ & $16.1 \pm 7.1$ \\
\hline $\begin{array}{l}\text { Tropidurus } \\
\text { etheridgei }\end{array}$ & Lagarto & $0.1 \pm 0.4$ & $1.1 \pm 1.2$ \\
\hline
\end{tabular}


Tabla 2. Estructura de la vegetación (media $\pm \mathrm{DE})$ de dos tipos de bosque del Chaco Semiárido. Estadístico de la prueba $t$ y valor de $P$ entre el Bosque Testigo $(\mathrm{n}=7)$ y Bosque con RBI ( $n=7)$. Ver descripción de los tipos de bosques en la leyenda de la Figura 1.

Table 2. Vegetation structure (mean \pm SD) of two types forest of Semiarid Chaco. $T$-test statistical and $P$ value between Untreated Forest (n=7) and Forest with RBI $(n=7)$. See description of forest types in the legend of Figure 1.

\begin{tabular}{ccccc}
\hline Variable & $\begin{array}{c}\text { Bosque } \\
\text { Testigo }\end{array}$ & $\begin{array}{c}\text { Bosque } \\
\text { con RBI }\end{array}$ & $t$ & $P$ \\
\hline $\begin{array}{c}\text { Cobertura } \\
\text { arbórea (\%) } \\
\begin{array}{c}\text { Cobertura } \\
\text { arbustiva (\%) } \\
\text { Cobertura }\end{array}\end{array}$ & $59.8 \pm 9.3$ & $47.6 \pm 7.4$ & 2.74 & 0.02 \\
$\begin{array}{c}\text { hojarasca (\%) } \\
\text { Cobertura residuos } \\
\text { leñosos finos (\%) }\end{array}$ & $13.8 \pm 6.9 \pm 11.0$ & $81.7 \pm 10.2$ & 0.83 & 0.42 \\
$\begin{array}{c}\text { Cobertura residuos } \\
\text { leñosos gruesos (\%) }\end{array}$ & $3.1 \pm 3.6$ & $13.9 \pm 5.7$ & -3.96 & 0.002 \\
$\begin{array}{c}\text { Cobertura } \\
\text { herbácea (\%) } \\
\text { Riqueza }\end{array}$ & $17.9 \pm 11.9$ & $20.6 \pm 12.8$ & 0.02 & 0.98 \\
$\begin{array}{c}\text { estructural } \\
\text { Diversidad } \\
\text { estructural }\end{array}$ & $5.7 \pm 0.5$ & $6.0 \pm 0.0$ & -1.55 & 0.17 \\
\hline
\end{tabular}

de la riqueza esperada de especies tuvo un valor medio de 9 (intervalo de confianza entre 7.2 y 28.9). En el Bosque con RBI, la riqueza observada de especies de reptiles fue 8 , y el estimador Chao1 de la riqueza esperada de

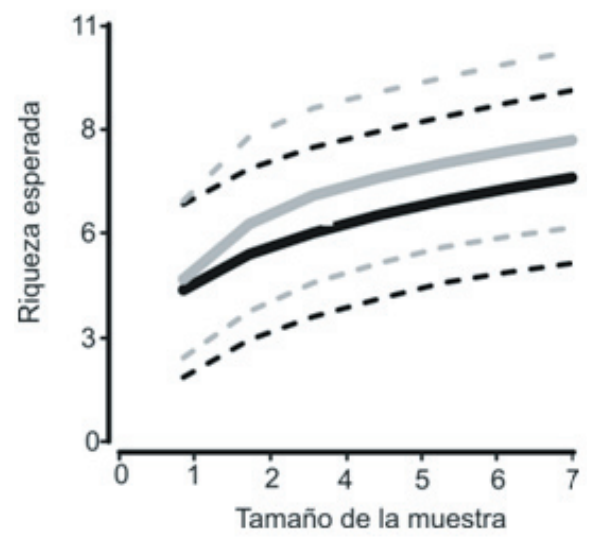

Figura 2. Riqueza de especies de reptiles de dos tipos de bosque del Chaco Semiárido, estimada mediante curvas de rarefacción usando el estimador Mao Tau. Bosque Testigo: riqueza esperada (línea sólida negra) e intervalos de confianza al 95\% (líneas punteadas negras). Bosque con RBI: riqueza esperada (línea sólida gris) e intervalos de confianza al 95\% (líneas punteadas grises). Bosque Testigo $(n=7)$ y Bosque con RBI (n=7). Ver descripción de los tipos de bosques en la leyenda de la Figura 1.

Figure 2. Reptile species richness in two forests types in Semiarid Chaco, estimated by rarefaction using the Mau Tau estimator. Untreated Forest: expected richness (solid black line) and $95 \%$ confidence interval (dashed black line). Forest with RBI: expected richness (solid grey line) and $95 \%$ confidence interval (dashed grey line). Untreated Forest $(n=7)$ and Forest with RBI $(n=7)$.See description of forest types in the legend of Figure 1. especies tuvo un valor medio de 10 (intervalo de confianza entre 8.2 y 30.0). Esto significa que el esfuerzo de muestreo aplicado detectó $77.8 \%$ de las especies potencialmente presentes en el Bosque Testigo y $80 \%$ en el Bosque con RBI. Los intervalos de confianza de las curvas de rarefacción de ambos hábitats se solaparon ampliamente (Figura 2), lo que indica que no existen diferencias significativas entre la riqueza de especies de los ensambles.

\section{Similitud estructural de los hábitats y composición de los ensambles de reptiles}

La similitud estructural de los tipos de bosques fue alta $(\mathrm{S}=85 \%)$. La similitud entre los ensambles de reptiles también fue alta $(\mathrm{CH}-\mathrm{J}=0.97 \pm 0.08)$.

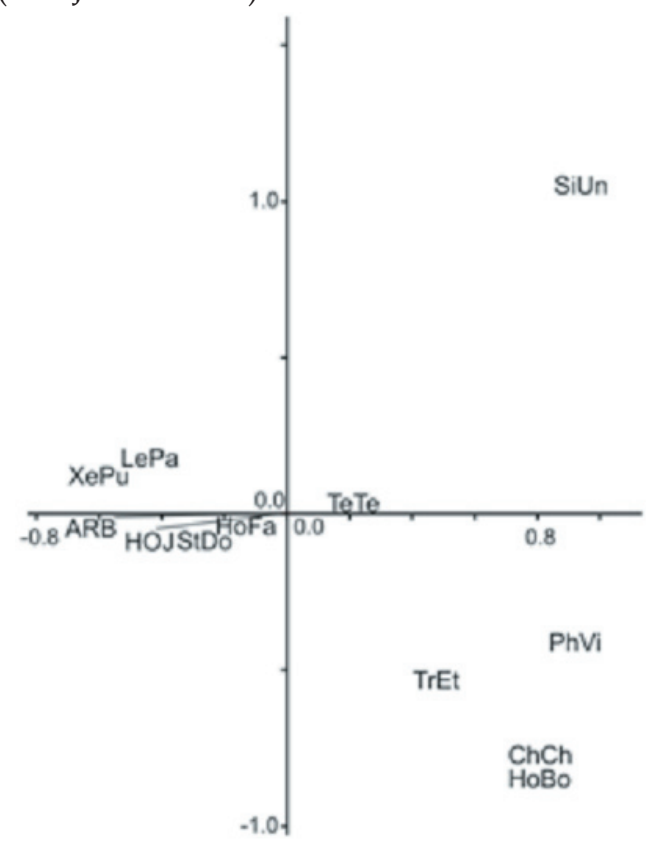

Figura 3. Análisis NMS (ejes 1 y 2) realizado sobre las abundancias de reptiles de dos tipos de bosques del Chaco Semiárido.Especies:Chelonoidischilensis(ChCh),Homonota borellii(HoBo),Homonotafasciata(HoFa),Leiosaurusparonae (LePa), Siagonodonun guirostris (SiUn), Xenodon pulcher (XePu),Phimophisvittatus(PhVi),Sternocercusdoellojuradoi (StDo), Teius teyou (TeTe) y Tropidurus etheridgei (TrEt). Variables de estructura de la vegetación: Cobertura arbórea (ARB) y Cobertura de hojarasca (HOJ). Bosque Testigo ( $n=7)$ y Bosque con RBI ( $n=7)$. Ver descripción de los tipos de bosques en la leyenda de la Figura 1.

Figure 3. NMS analysis (axes 1 and 2) on reptile abundances of two type forests of Semiarid Chaco. Species: Chelonoidis chilensis (ChCh), Homonota borellii (HoBo), Homonota fasciata (HoFa), Leiosaurus paronae (LePa), Siagonodon unguirostris (SiUn), Xenodon pulcher (XePu),Phimophisvittatus(PhVi),Sternocercusdoellojuradoi (StDo), Teius teyou (TeTe) y Tropidurus etheridgei (TrEt). Vegetation structure variables: Tree cover (ARB) and Litter cover (HOJ). Untreated Forest $(n=7)$ and Forest with RBI $(n=7)$. See description of forest types in the legend of Figure 1. 
El NMS sobre las abundancias de reptiles resultó en una solución óptima de 3 ejes que redujo significativamente el estrés (prueba de Monte Carlo, $P=0.012$; estrés $=4.8$; inestabilidad $=0$ ). El eje 1 del NMS representó $58 \%$ de la variabilidad total; el eje 2, 20.8\% y el eje 3, el 16.8\%. Las variables ambientales de mayor importancia fueron la cobertura arbórea y la cobertura de hojarasca, que también mostraron una asociación estrecha con el eje 1 (Figura 3). El eje 1 puede interpretarse como un gradiente de luz solar donde las especies que se posicionan a la izquierda del gráfico requieren ambientes más sombreados (e.g., Sternocercus doellojuradoi) y las que se ubican a la derecha requieren ambientes con mayor insolación a nivel del suelo (e.g., Teius teyou). Los ejes 2 y 3 no reflejaron ningún gradiente ambiental subyacente claro.

\section{DisCUSIÓN}

\section{Complejidadestructuralverticaldelavegetación $y$ diversidad alfa de reptiles}

La vegetación estructuralmente compleja tiende a soportar mayor biodiversidad que la vegetación estructuralmente simple o degradada (Fischer et al. 2006) debido a que puede proveer más nichos y diversidad de maneras de explotar los recursos ambientales (Tews et al. 2004). Pianka (1967) reportó una relación positiva entre la diversidad y la heterogeneidad del hábitat para reptiles de desierto en Norte América. Pelegrin and Bucher (2012) en el Chaco Árido reportaron que la diversidad y abundancia de lagartos fue más alta en hábitats de mayor complejidad vertical, como bosques primario y moderadamente degradado, que en un pastizal generado por fuego y en un bosque severamente degradado. Este estudio muestra que en el bosque con RBI: a) no disminuyó la riqueza de reptiles, b) se mantuvo la riqueza estructural de la vegetación, y c) a pesar de una reducción moderada de la cobertura arbórea y arbustiva, se incrementó la diversidad estructural gracias a sustanciales incrementos de la cobertura de residuos leñosos. Estos resultados apoyan nuestra predicción de que el RBI no degrada la estructura vertical de la vegetación por lo que no impacta negativamente en la diversidad alfa de reptiles. Nuestros resultados se condicen con el estudio de Kunst et al. (2016), quienes observaron que la diversidad alfa de reptiles y anfibios en bosques no disminuye al año de ser tratados con RBI sin siembra de pasturas exóticas. Ese trabajo es el único antecedente previo referido a las relaciones entre el RBI y la herpetofauna.

\section{Similitud estructural de los hábitats y composición de los ensambles de reptiles}

La estructura del paisaje, la intensidad de uso de la tierra y las similitudes entre los hábitats pueden influenciar las similitudes entre las comunidades ecológicas (Dormann et al. 2007). Diversos estudios mostraron que el RBI ejerce un impacto bajo sobre la vegetación y genera hábitats estructural y florísticamente similares a los bosques sin tratar (Kunst et al. 2008; Albanesi et al. 2013; Coria et al. 2015). En este estudio, los valores obtenidos de S (85\%) y CH-J (0.97) apoyan nuestra predicción acerca de que el bosque con RBI fue estructuralmente similar al bosque sin disturbar. En relación con este resultado, las composiciones de los ensambles de reptiles también fueron similares entre ambos hábitats. Fischer et al. (2006) sostienen que los hábitats antropizados y con estructuras similares a la de los parches de hábitat de referencia no sólo proveen hábitats permanentes para especies animales, sino que también otorgan mayor conectividad al paisaje y reducen los efectos de borde. Sobre la base de las afirmaciones de Fischer et al. (2006), proponemos que las condiciones estructurales del bosque con RBI mantuvieron los microhábitats específicos para las especies reptiles y no impusieron mayores restricciones al intercambio de especies con los bosques circundantes, incidiendo en la alta similitud observada entre los ensambles.

Las variables de estructura de la vegetación más importantes para los ensambles de reptiles fueron la cobertura arbórea y la cobertura de hojarasca, que pueden interpretarse como asociadas a un gradiente ambiental de luz solar que influye en la distribución de las abundancias de las especies. En el bosque con RBI, la cobertura arbórea se redujo $20.4 \%$ (mayormente debido al aprovechamiento forestal) y la cobertura de hojarasca permaneció sin variación. Se ha sugerido que en el Chaco Semiárido, la selección de microhábitats por lagartos es un proceso influenciado por la estructura de la vegetación y que probablemente esté regulado por los requerimientos térmicos de las especies (Pelegrin et al. 2009). En este estudio, las abundancias de las especies de lagartos reflejaron dicho gradiente ambiental de luz solar. Homonota fasciata, Leiosaurus paronaey Sternocercus doellojuradoi son especialistas de bosque que requieren ambientes sombreados 
para vivir (Leynaud and Bucher 2005; Pelegrin and Bucher 2012) y mostraron una asociación positiva y estrecha con las coberturas arbórea y de hojarasca (i.e., mayor sombra). En cambio, Teius teyou y Tropidurus etheridgei prefieren ambientes más abiertos, con suelos desnudos y mayor insolación (Leynaud and Bucher 2005; Pelegrin and Bucher 2012) y no mostraron una asociación con las coberturas arbórea y de hojarasca. Las especies especialistas de bosque mantuvieron en el bosque con RBI similares abundancias a las halladas en el bosque sin disturbar, lo que indica la potencialidad del RBI para conservar a este grupo sensible de especies.

\section{Conclusiones y necesidades de estudios adicionales}

La diversidad alfa de especies no siempre es suficiente para evaluar las prácticas de manejo de la tierra dado que el intercambio de especies entre las comunidades locales también puede verse afectado. Por ello, la similitud entre las comunidades puede ser una medida útil de la integridad de los ecosistemas (Dormann et al. 2007). Este estudio muestra que la diversidad alfa de reptiles no disminuye en el bosque con RBI (dado que la estructura vertical de la vegetación no fue degradada) y que el ensamble de reptiles del bosque con RBI fue altamente similar al del bosque sin disturbar (asociado a una alta similitud estructural de la vegetación entre estos hábitats). A escala local, el RBI sin siembra de pasturas exóticas tiene potencial para conservar adecuadamente la diversidad y composición de los ensambles de reptiles de bosques del Chaco Semiárido. Sin embargo, se necesitan estudios con intensidades de muestreo mayores y en otros contextos de paisaje (e.g., fragmentados) para arribar a conclusiones más generalizadas acerca de las relaciones entre el RBI y los ensambles de reptiles. Además, puesto que la introducción de pasturas exóticas puede disminuir la heterogeneidad estructural del estrato herbáceo y reducir la riqueza y abundancia de reptiles (Abom et al. 2015), se requieren estudios adicionales para evaluar la influencia de la siembra de pasturas exóticas (típicamente Megathyrsus maximus cv. gatton panic) sobre los ensambles de reptiles en bosques tratados con RBI.

Agradecimientos. A María Eugenia Periago (Fundación Vida Silvestre Argentina) por la corrección del Abstract en idioma inglés. La presente investigación fue financiada por el Proyecto de Investigación PNNAT-1128053 "Evaluación del impacto de las actividades productivas sobre la biodiversidad y sus servicios ecosistémicos" del INTA, Argentina.

\section{REFERENCIAS}

Abom, R., W. Vogler, and L. Schwarzkopf. 2015. Mechanisms of the impact of a weed (grader grass, Themedaquadrivalvis) on reptile assemblage structure in a tropical savannah. Biol. Conserv. 191:75-82. DOI: 10.1016/j.biocon.2015.06.016.

Albanesi, A., C. R. Kunst, A. L. Anriquez, J. E. Silverman, and R. Ledesma. 2013. Rolado selectivo de baja intensidad (RBI) y sistemas silvopastoriles de la región chaqueña. Pages 147-174 in A. Albanesi, R. Paz, M. T. Sobrero, S. Helman, and S. Rodriguez (eds.). Hacia la Construcción del desarrollo agropecuario y agroindustrial. De La FAyA Al NOA. Ediciones Magna, Tucumán, Tucumán, Argentina.

Boletta, P. 1998. Clima. Pages 7-21 in R. Casas (ed.). Desmonte y habilitación de tierras en la región chaqueña semiárida. FAO. Santiago, Región Metropolitana de Chile, Chile.

Cano, P. D., and G. C. Leynaud. 2009. Effects of fire and cattle grazing on amphibians and lizards in northeastern Argentina (Humid Chaco). Eur. J. Wildl. Res. 56:411-420. DOI:10.1007/s10344-009-0335-7.

Chao, A., R. L. Chazdon, R. K. Colwell, and T. J. Shen. 2005. A new statistical approach for assessing compositional similarity based on incidence and abundance data. Ecol. Lett. 8:148-159. DOI: 10.1111/j.1461-0248.2004.00707.x.

Clarke, K. R., and R. M. Warwick. 2001. Change in marine communities: an approach to statistical analysis and interpretation. Second edition. PRIMERE, Plymouth, Devon, UK.

Colwell, R. K. 2013. EstimateS: Statistical estimation of species richness and shared species from samples. Version 9. User's Guide. <http:/ / purl.oclc.org/estimates>. Downloaded on 1 June 2016.

Coria, R. D., O. R. Coria, and C. R. Kunst. 2015. Influencia del rolado selectivo de baja intensidad (RBI) sobre las comunidades de aves de bosques del Chaco Occidental. Ecol. Austral 25:158-171.

Coria, R. D., O. R. Coria, and C. R. Kunst. 2016. Diversidad y composición de especies de aves en un gradiente BosqueArbustal-Sabana del Chaco Semiárido, Argentina. Ornitol. Neotropical 27:1-15.

Di Rienzo, J. A., F. Casanoves, L. A. González, E. M. Tablada, M. P. Díaz, C. Robledo, and M. G. Balzarini. 2008. Estadística para las Ciencias Agropecuarias. Seventh edition. Editorial Brujas, Córdoba, Córdoba, Argentina.

Di Rienzo, J. A., F. Casanoves, M. G. Balzarini, L. González, M. Tablada, and C. Robledo. 2013. Infostat versión 2013. Grupo Infostat, FCA, Universidad Nacional de Córdoba, Córdoba, Córdoba, Argentina.

Dormann, C. F., O. Schweiger, I. Augenstein, D. Bailey, R. Billeter, G. de Blust, R. DeFilippi, M. Frenzel, F. Hendrickx, F. Herzog, S. Klotz, J. Liira, J. P. Maelfait, T. Schmidt, M. Speelmans, W. K. R. E. van Wingerden, and M. Zobel. 2007. Effects of landscape structure and land-use intensity on similarity of plant and animal communities. Glob. Ecol. 
Biogeogr. 16:774-787. DOI: 10.1111/j.1466-8238.2007.00344.x.

Fischer, J., D. B. Lindenmayer, and A. D. Manning. 2006. Biodiversity, ecosystem function, and resilience: ten guiding principles for commodity production landscapes. Front. Ecol. Environ. 4:80-86. DOI: 10.1890/1540-9295(2006)004[0080: BEFART]2.0.CO;2.

Fitzgerald, L. A., F. B. Cruz, and G. Perotti. 1999. Phenology of a lizard assemblage in the Dry Chaco of Argentina. J. Herpetol. 33:526-535.

Kunst, C., E. Monti, H. Pérez, and J. Godoy. 2006. Assessment of the rangelands of southwestern Santiago del Estero, Argentina, for grazing management and research. J. Environ. Manage. 80:248-265. DOI:10.1016/ j.jenvman.2005.10.001.

Kunst, C., M. Navall, R. D. Coria, R. Ledesma, P. Tomsic, A. González, A. Gómez, and D. Feullidae. 2015a. Guía de prácticas recomendables para sistemas silvopastoriles en Santiago del Estero. Producir carne y madera conservando el ambiente. INTA EEA Santiago del Estero, Gobierno de la Provincia de Santiago del Estero, Santiago del Estero, Santiago del Estero, Argentina.

Kunst, C., M. Navall, R. Ledesma, J. Silberman, A. Anriquez, D. Coria, S. Bravo, A. Gómez, A. Albanesi, D. Grasso, J. A. Dominguez, A. González, P. Tomsic, and J. Godoy. 2016. Silvopastoral Systems in the Western Chaco Region, Argentina. Pages 3-39 in P. Peri, F. Dube, and F. Varella (eds.). Silvopastoral systems in southern South America, Advances in Agroforestry 11. Springer International Publishing Switzerland, New York, New York, USA. DOI: 10.1007/978-3-319-24109-8_4.

Kunst, C., R. Ledesma, and M. Navall (eds.). 2008. RBI: Rolado Selectivo de Baja Intensidad. INTA. Santiago del Estero, Santiago del Estero, Argentina.

Kunst, C., R. Ledesma, M. Navall, A. Gómez, D. Coria, J. Arroquy, M. Ávila, P. Tomsic, A. González, A. Albanesi, J. Silberman, A. Anriquez, and S. Bravo. 2015b. RBI: Rolado Selectivo de Baja Intensidad. INTA EEA Santiago del Estero, Gobierno de la Provincia de Santiago del Estero, Santiago del Estero, Santiago del Estero, Argentina.

Lescano, J. N., L. M. Bellis, L. E. Hoyos, and G. C. Leynaud. 2015. Amphibian assemblages in dry forests: multi-scale variables explain variations in species richness. Acta Oecol. 65-66:41-50. DOI: 10.1016/j.actao.2015.05.002.

Leynaud, G. C., and E. H. Bucher. 2001. Composition, relative abundance and activity patterns of the snake fauna of Los Colorados Biological Station (Western Chaco of Argentina). Boletin Acad. Nac. Cienc. 66:125-132.

Leynaud, G. C., and E. H. Bucher. 2005. Restoration of degraded Chaco woodlands: effects on reptile assemblages. For. Ecol. Manag. 213:384-390. DOI:10.1016/j.foreco.2005.04.003.

Mastrangelo, M. E., and M. C. Gavin. 2012. Trade-offs between cattle production and bird conservation in an agricultural frontier of the Gran Chaco of Argentina. Conserv. Biol. 26:1040-1051. DOI: 10.1111/j.1523-1739.2012.01904.x.

Mccune, B., and M. J. Mefford. 2011. PC-ORD. Multivariate analysis of ecological data. Version 6.15. MjM Software Design, Gleneden Beach, Oregon, USA.

Mccune, B., J. B. Grace, and D. L. Urban. 2002. Analysis of ecological communities. MjM Software Design, Gleneden Beach, Oregon, USA. 